\title{
The Influence of Ultraviolet Illumination on the Passive Behavior of Zinc
}

\author{
Amy L. Rudd and Carmel B. Breslin*,z \\ Department of Chemistry, National University of Ireland Maynooth, County Kildare, Ireland
}

\begin{abstract}
The passive behavior of zinc in alkaline $\mathrm{pH}$ 13.0, 10.3, and 9.2 solutions, under dark and illumination conditions, has been studied using dc polarization and ac impedance techniques. It was found that illumination with polychromatic light caused dissolution of anodically formed passive layers on zinc, with this photoinduced dissolution effect being more pronounced in the more alkaline $\mathrm{pH} 13.0$ solution. Photoinduced dissolution in the $\mathrm{pH} 10.3$ and 9.2 solutions is explained in terms of the photodecomposition of $\mathrm{ZnO}$ through reaction with photogenerated holes. The more intense dissolution in the $\mathrm{pH} 13.0$ solution is explained in terms of the photodecomposition of $\mathrm{ZnO}$ and the additional electrochemical dissolution in this alkaline solution which occurs as a result of the photodecomposition of the passive layer. Although the electrodes repassivated once the light source was removed, it was found that prior illumination led to a decrease in the donor densities and thus a modification of the defect structure of the passive layers.
\end{abstract}

(C) 2000 The Electrochemical Society. S0013-4651(99)08-104-5. All rights reserved.

Manuscript submitted August 26, 1999; revised manuscript received December 12, 1999.

Zinc and many zinc alloys are used widely as metallic coatings in the corrosion protection of iron and steel. These systems exhibit passive behavior in alkaline solutions due to the formation of a zinc oxide/hydroxide passive film. ${ }^{1,2}$ This passive layer possesses some properties similar to that of the nonstoichiometric $\mathrm{ZnO},{ }^{3-6}$ which is an n-type semiconductor with a large optical bandgap energy of approximately $3.2 \mathrm{eV}$. 7,8

This nonstoichiometric $\mathrm{ZnO}$ is well known as a photoelectrochemical electrode. On illumination of the anodically biased $\mathrm{ZnO}$ with sufficiently energetic photons, electrons are promoted from the valence to the conduction band generating electron-hole pairs. The holes are transported to the film-solution interface where they undergo an electrochemical reaction, either oxidizing suitable chemical species added to the electrolyte, or causing photodissolution of $\mathrm{ZnO} .{ }^{9-11}$ Indeed, this photoinduced dissolution of $\mathrm{ZnO}$ can be used for micropattern processing. ${ }^{12,13}$

However, the influence of illumination on the passive and corrosion behavior of pure zinc or zinc-rich electrodes or coatings is less well documented, with only a few reports appearing in the literature. For example, Juzeliunas et al. ${ }^{14}$ reported an increase in the corrosion rate of zinc in a $5 \% \mathrm{NaCl}$ solution on illumination at $488 \mathrm{~nm}$. This increase in the corrosion rate was associated with a light-induced increase in the rate of the oxygen reduction reaction, but no changes in the anodic polarization behavior were observed. Spathis and Poulios ${ }^{15}$ observed the photocorrosion of zinc and zinc oxide coatings in a $3.5 \% \mathrm{NaCl}$ solution on illumination with white light.

In this communication the results of an investigation into the influence of ultraviolet (UV) illumination on the passive behavior of $\mathrm{Zn}$ in alkaline and slightly alkaline solutions are presented.

\section{Experimental}

Test electrodes were prepared from high purity zinc rods $\left(99.999^{+} \%\right)$. Electrical contact with the zinc rod samples was achieved by means of a copper wire threaded into the base of the metal sample. This entire unit was then sealed in a Teflon holder, and the exposed surface of the zinc rod was set with epoxy resin in the Teflon sleeve. Prior to each test the samples were polished with successively finer grades of $\mathrm{SiC}$ paper to a smooth surface finish and rinsed with distilled water. In the case of the Mott-Schottky experiments the electrodes were polished to a mirror finish using alumina powder. The electrochemical cell was made of Teflon with a quartz window in the base to allow irradiation of the test electrodes. Highdensity graphite rods were used as the auxiliary electrodes and a saturated calomel electrode (SCE) was used as the reference electrode. All potentials quoted are relative to this electrode. The electrolytes were prepared using analytical grade reagents and distilled water.

\footnotetext{
* Electrochemical Society Active Member.
}

z E-mail: cb.breslin@may.ie
The electrolytes used were an alkaline $\mathrm{pH} 13.0$ borate solution (0.1 mol dm ${ }^{-3} \mathrm{NaOH} / 0.025 \mathrm{~mol} \mathrm{dm}-3 \mathrm{Na}_{2} \mathrm{~B}_{4} \mathrm{O}_{7}$ ) and a slightly alkaline, $\mathrm{pH} 10.3\left(0.042 \mathrm{~mol} \mathrm{dm}^{-3} \mathrm{NaOH} / 0.025 \mathrm{~mol} \mathrm{dm}^{-3}\right.$ $\left.\mathrm{Na}_{2} \mathrm{~B}_{4} \mathrm{O}_{7}\right)$ and $\mathrm{pH}$ 9.2, borate electrolyte $\left(0.001 \mathrm{~mol} \mathrm{dm}^{-3}\right.$ $\mathrm{NaOH} / 0.025 \mathrm{~mol} \mathrm{dm}^{-3} \mathrm{Na}_{2} \mathrm{~B}_{4} \mathrm{O}_{7}$ ).

A $300 \mathrm{~W}$ xenon arc lamp (Oriel model 6258) was used as the illumination source. The light was passed through a water cooler to remove infrared radiation. The beam was then passed through a series of filters, lens, and mirrors and focused on the stage to illuminate the entire surface of exposed electrode. The intensity of the light entering the cell was measured as $250 \mathrm{~mW} / \mathrm{cm}^{2}$ for unfiltered irradiation using a Spectra-Physics CW laser power meter model 407A. A maximum temperature rise of $1.9^{\circ} \mathrm{C}$ was recorded over a 30 min illumination period with this high intensity polychromatic illumination procedure.

Potentiodynamic electrochemical experiments were carried out using an EG\&G potentiostat model 263. The working electrodes were polarized from the corrosion potential at a scan rate of $0.5 \mathrm{mV}$ $\mathrm{s}^{-1}$ in the anodic direction, following an initial delay period of $1.3 \times$ $10^{3} \mathrm{~s}$ under open-circuit conditions. Impedance measurements were recorded using a Solartron 1250 frequency response analyzer and an EI 1287 electrochemical interface. The spectra were recorded at some applied potential. An excitation voltage of $10 \mathrm{mV}$ was used. All impedance data were fit to appropriate equivalent circuits using a complex nonlinear least squares fitting routine, using both the real and imaginary components of the data. Mott-Schottky analyses were performed by measuring the capacitance as a function of potential at $1 \mathrm{kHz}$ using an excitation voltage of $10 \mathrm{mV}$ and a sweep rate of $0.01 \mathrm{~V} \mathrm{~s}^{-1}$. Capacitance-voltage data were recorded using a $10 \mathrm{mV}$ excitation voltage and a sweep rate of $5 \mathrm{mV} \mathrm{s}^{-1}$.

\section{Results}

Photoinduced dissolution.-The polarization behavior of zinc in alkaline $\mathrm{pH} 13.0$ and 9.2 solutions under continuous conditions of illumination and nonillumination is shown in Fig. 1. These measurements were performed by first allowing the zinc electrode to stabilize in the solution for $1.3 \times 10^{3} \mathrm{~s}$ and then polarizing the electrode in the dark or under continuous illumination from the corrosion potential. These data are somewhat different to those reported in other communications ${ }^{2,6}$ as a prereduction step was not employed. Thus, the high anodic currents normally observed in the potential region of $-1.2 \mathrm{~V}$ (SCE) are not seen here. The polarization plots obtained under dark conditions are characterized by passive regions in the potential intervals of $-600 \mathrm{mV}(\mathrm{SCE})$ to about 1.0 V(SCE). However, the passive current densities are close to an order of magnitude higher in the more alkaline $\mathrm{pH} 13.0$ solution. At potentials more anodic than about $1.2 \mathrm{~V}$, oxygen evolution occurs giving rise to the relatively sharp increase in current. It can be seen from these data that continuous illumination gives rise to larger anodic currents in both solutions, however the photoin- 


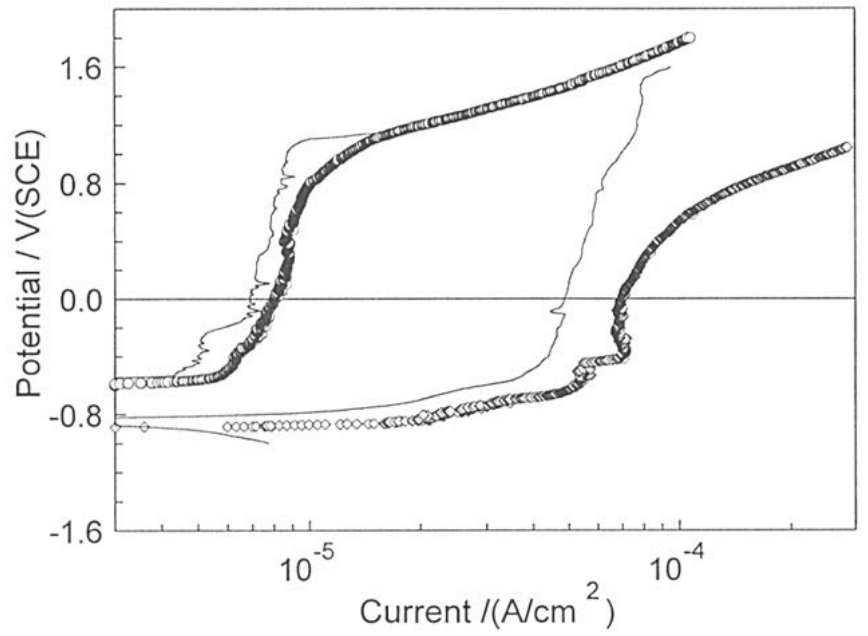

Figure 1. Anodic polarization plots recorded for Zn under (-) (low current densities) dark and $(\bigcirc)$ illumination conditions in $\mathrm{pH} 9.2$ solution, (-) (high current densities) dark and $(\diamond)$ illumination conditions in $\mathrm{pH} 13.0$ solution.

duced currents are greater in the $\mathrm{pH} 13.0$ solution. It is also evident from these plots that the photoinduced currents are higher at higher applied potentials. This is clearly evident for the data obtained in the alkaline $\mathrm{pH} 13.0$ solution.

In Fig. 2a, the influence of a light stimulus on the anodic behavior of zinc in the alkaline $\mathrm{pH} 9.2$ and 10.3 solutions is shown while similar data recorded over different time periods are shown in Fig. $2 b$ for the alkaline $\mathrm{pH} 13.0$ solution. In these tests the electrodes were polarized at $800 \mathrm{mV}(\mathrm{SCE})$ in the alkaline solutions. In the case
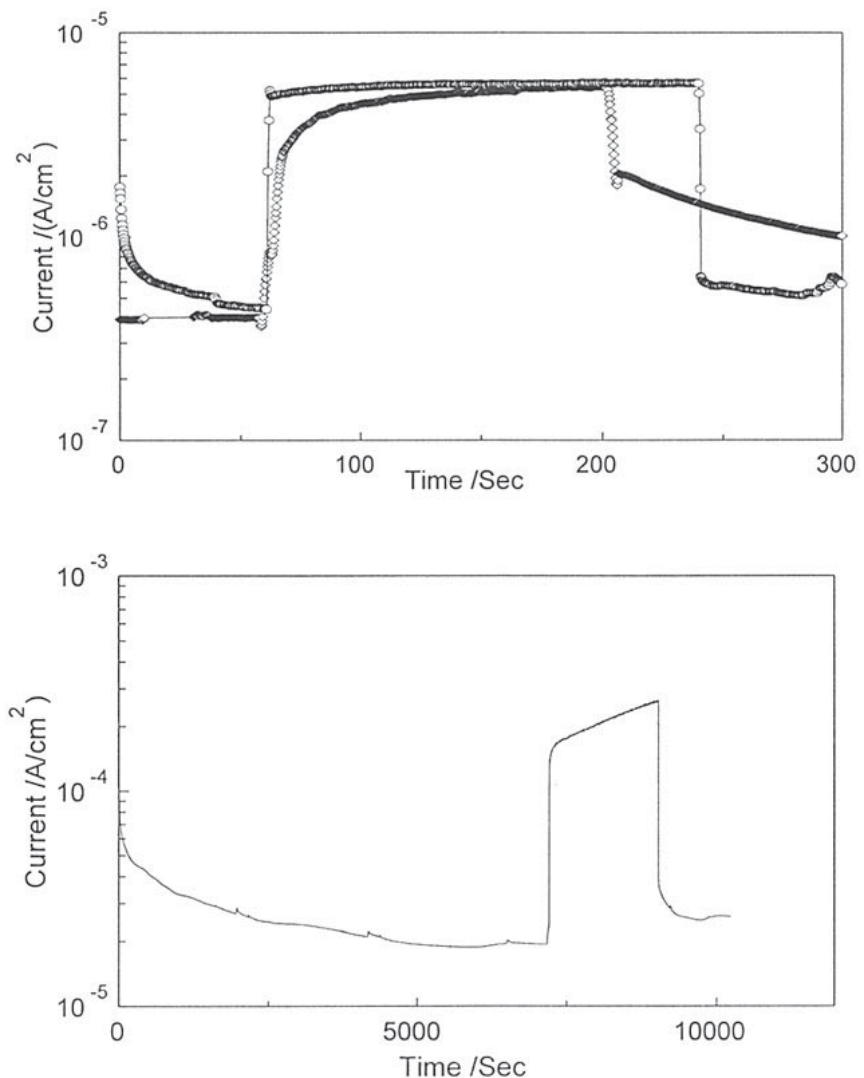

Figure 2. Potentiostatic current-time plots recorded for $\mathrm{Zn}$ in (a, top) $\mathrm{pH} 9.2$ $(\bigcirc)$ and $\mathrm{pH} 10.3(\diamond)$ electrolytes; (b, bottom) pH 13.0 electrolyte; light turned on at point where current increases sharply and removed at point where current decreases sharply. of the $\mathrm{pH} 9.2$ and 10.3 solutions the surface was illuminated after a total passivation period of $15.5 \mathrm{~h}$, but only the data recorded close to the illumination period are shown and are scaled with respect to zero time. Illumination was continued for a further period of approximately $180 \mathrm{~s}$, at which point the light was removed. Within a few seconds of illumination, a sharp increase in the current was observed, and once the light stimulus was removed the surface repassivated, Fig. 2a. However, it can be seen, particularly in the case of the $\mathrm{pH} 10.3$ solution, that the passive current does not return to the exact value prior to illumination, suggesting some change in the passive behavior of the electrode. The data shown in Fig. $2 \mathrm{~b}$ for the $\mathrm{pH} 13.0$ electrolyte were recorded after a $2 \mathrm{~h}$ passivation period. Again, on illumination of the surface a sharp increase in the anodic current was observed. In this case, the current continued to increase during the illumination period, signifying increasingly intense dissolution. Furthermore there was a difference of about $5 \mu \mathrm{A} \mathrm{cm}^{-2}$ in the current before and following the illumination period. This tends to suggest that the intense dissolution observed during the 30 min illumination period leads to some degree of surface roughening and a change in the surface area. It can be seen from a comparison of Fig. 2a and b that the photocurrents observed under these strong alkaline conditions are considerably higher than those recorded in the $\mathrm{pH} 9.2$ and 10.3 solutions, suggesting considerably higher photoinduced dissolution of the zinc electrode in the alkaline $\mathrm{pH} 13.0$ solution.

The steady-state photocurrents measured as a function of the passivation potential for $\mathrm{Zn}$ polarized in the $\mathrm{pH} 9.2$ and 10.3 solutions are shown in Fig. 3a. These data were recorded by measuring the steady-state photo currents (obtained after approximately $120 \mathrm{~s}$ ) following a $15.5 \mathrm{~h}$ illumination period at different polarization potentials. It can be seen, in agreement with the anodic polarization data, that the anodic photocurrents are significantly higher under high anodic bias, and that there is very little difference in the magnitude of the photocurrents in the $\mathrm{pH} 10.3$ and 9.2 solutions. In Fig. 3b, the steady-state anodic photocurrents recorded at $800 \mathrm{mV}$ (SCE) follow-
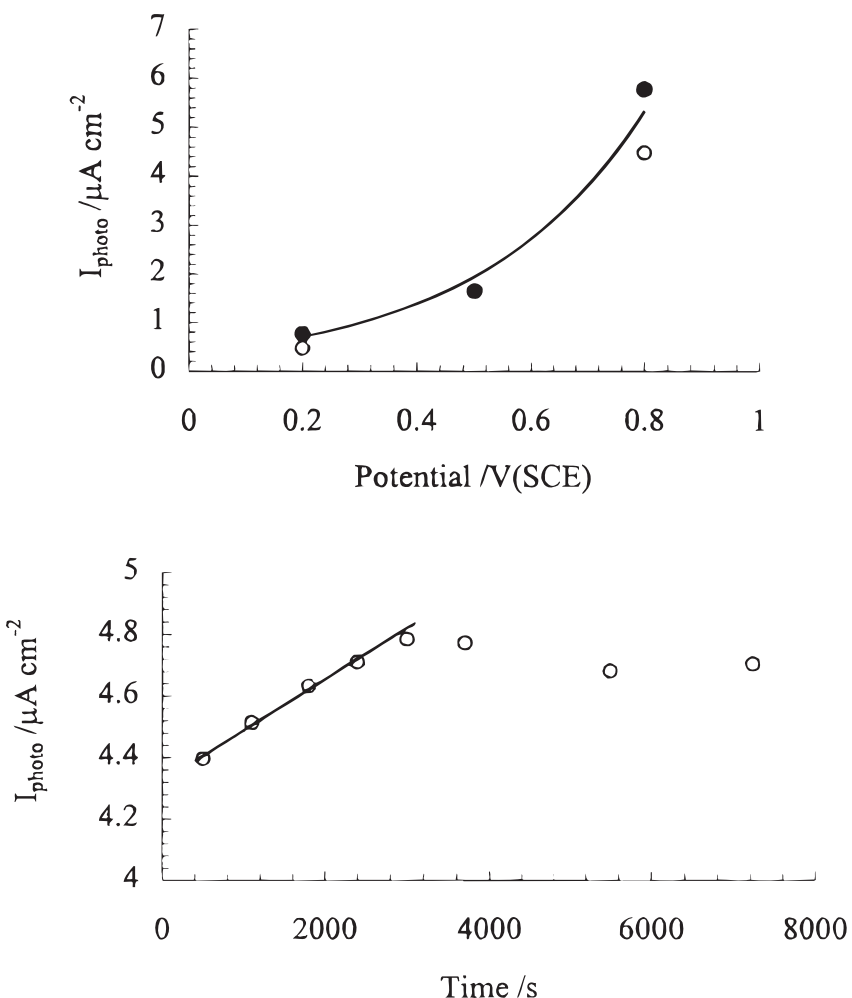

Figure 3. (a, top) Steady-state photocurrent plotted as a function of the formation potential for $\mathrm{Zn}$ polarized in $\mathrm{pH} 9.2(\bigcirc)$ and $\mathrm{pH} 10.3(\bigcirc)$ solutions. (b, bottom) Steady-state photocurrent as a function of the formation time for $\mathrm{Zn}$ polarized at $+800 \mathrm{mV}(\mathrm{SCE})$ in $\mathrm{pH} 9.2$ solution. 
ing different passivation periods are shown for the $\mathrm{pH} 9.2$ solution. Here, it is seen that the anodic photocurrents increase linearly with the passivation period for the first 40 min of polarization. This is consistent with the growth of the passive film and the formation of the semiconducting $\mathrm{ZnO}$ phase during the early stages of polarization.

In Fig. 4, 5, and 6 impedance data recorded for zinc in the $\mathrm{pH}$ $10.3,13.0$, and 9.2 solutions during polarization at $800 \mathrm{mV}$ (SCE) under conditions of illumination and nonillumination are shown. In these plots the symbols represent the experimental data, while the solid lines represent the theoretical data generated using the circuits depicted in these figures. In Fig. 4a the impedance data are shown for the $\mathrm{pH} 10.3$ solution under three different sets of conditions. In these cases, the electrodes were first polarized at $800 \mathrm{mV}$ (SCE) for $15 \mathrm{~h}$ in the dark to ensure the formation of a stable passive layer, then the impedance data were recorded at $800 \mathrm{mV}$ (SCE) in the dark. The surface was then illuminated, and the impedance data were recorded during the illumination conditions. The light source was then removed and the impedance data were recorded again under dark conditions. It can be seen in this case that illumination of the surface has a significant effect on the impedance data, decreasing the impedance at low frequencies, and altering the capacitive region. On remeasuring the impedance data after the illumination period has elapsed, the data obtained, although slightly different from those originally recorded under dark conditions, show evidence of the system returning to its passive state.

As evident in Fig. 4b two different equivalent circuits, one for dark conditions and one for light conditions, were used to fit the impedance data. Here, $R$ represents resistance elements, with $R_{\mathrm{s}}$ representing the solution resistance and $R_{\mathrm{CT}}$ representing the chargetransfer resistance while $Q_{1}$ and $Q_{2}$ are constant phase elements.
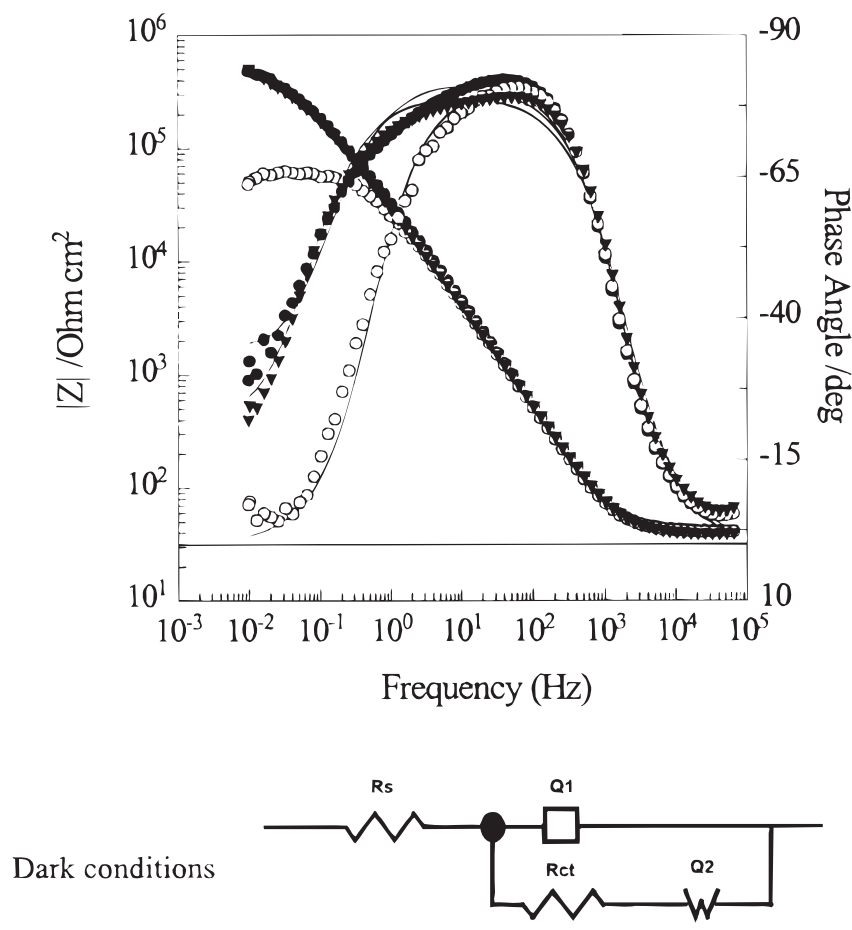

Q1

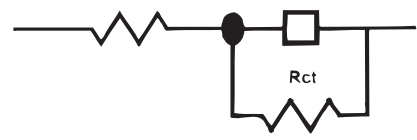

Figure 4. (a, top) Impedance data recorded under initial dark conditions ( $)$, followed by continuous illumination conditions $(\bigcirc)$, followed by dark conditions $(\boldsymbol{\nabla})$ for Zn polarized at $800 \mathrm{mV}$ (SCE) in $\mathrm{pH}$ 10.3. (b) Equivalent circuits used in the fitting of impedance data.
Very good agreement between the experimental data and the theoretical fitted data was obtained when these frequency-dependent constant-phase elements (CPE) were used as opposed to using pure capacitance and Warburg impedances. The impedance of a constantphase element, is defined as $Z_{\mathrm{CPE}}=\left[Q(j \omega)^{n}\right]^{-1}$ where $-1 \leq n \leq$ $1 .{ }^{16}$ Depending on the value of $n$, then the constant-phase element may represent a capacitor if $n$ is unity, or a Warburg diffusion element if $n$ is 0.5 . In the case of the data recorded in the dark, Fig. 4b, $Q_{1}$, with an $n$ value close to unity, represents the capacitance of the passive film, which may be taken to be equivalent to the capacitance of the space-charge layer. $Q_{2}$, with an $n$ value close to 0.5 , indicates a Warburg diffusion term, and represents the diffusional processes associated with the passive film. In this circuit analysis a separate series RC combination representing the Helmholtz layer was not used, in order to minimize the number of circuit elements, but instead is incorporated in the $Q_{1} R_{\mathrm{CT}}$ couple. But, because the Helmholtz capacitance is an order of magnitude higher than the passive film capacitance, then $Q_{1}$ may be taken to represent the capacitance of the passive film. There was no evidence of any diffusion process for the specimens polarized under continuous illumination conditions. In this case, a simple parallel RC circuit, with the $n$ value for $Q_{1}$ close to unity, was used to fit the data. This indicates that under illumination conditions a faradaic reaction takes place with an impedance for charge-transfer of $R_{\mathrm{CT}}$.

A similar photoinduced dissolution effect can be seen for the data recorded in the pH 13.0 and 9.2 solutions, Fig. 5 and 6, respectively. These data were recorded following a passivation period of $15 \mathrm{~h}$ for the $\mathrm{pH} 9.2$ solution and $2 \mathrm{~h}$ for the $\mathrm{pH} 13.0$ solution. These different time periods were used because longer passivation periods in the $\mathrm{pH}$ 13.0 solution lead to destabilization of the passive layer. Again, the first impedance measurements were made under dark conditions, the

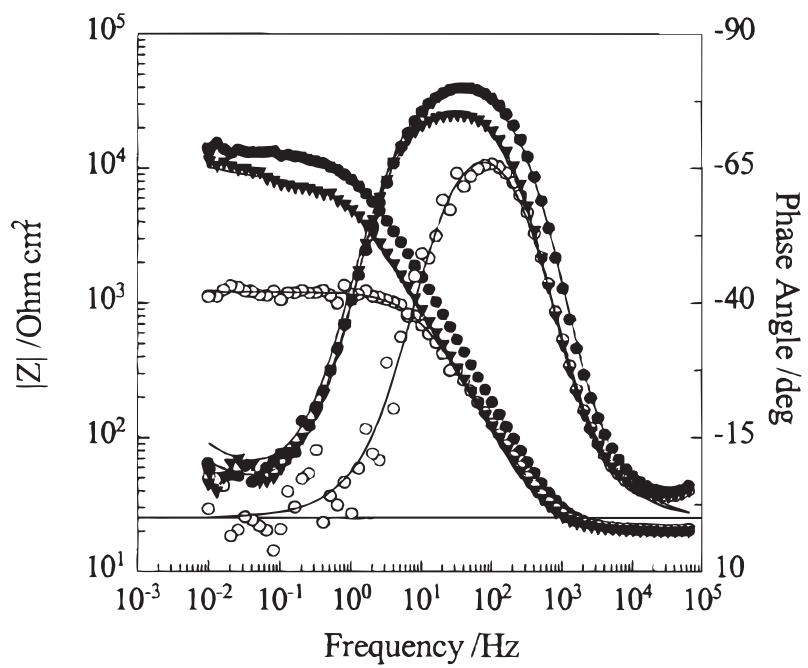

Dark conditions

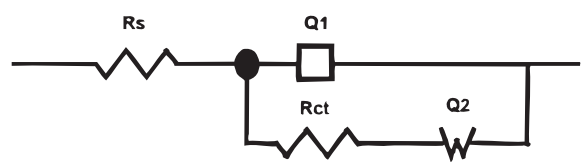

Light conditions

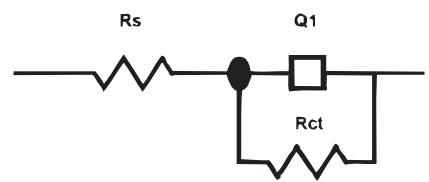

Figure 5. (a, top) Impedance data recorded under initial dark conditions $(\mathbf{\bullet})$ followed by continuous illumination conditions $(\bigcirc)$, followed by dark conditions $(\boldsymbol{\nabla})$ for $\mathrm{Zn}$ polarized at $800 \mathrm{mV}(\mathrm{SCE})$ in $\mathrm{pH}$ 13.0. (b) Equivalent circuits used in the fitting of impedance data. 

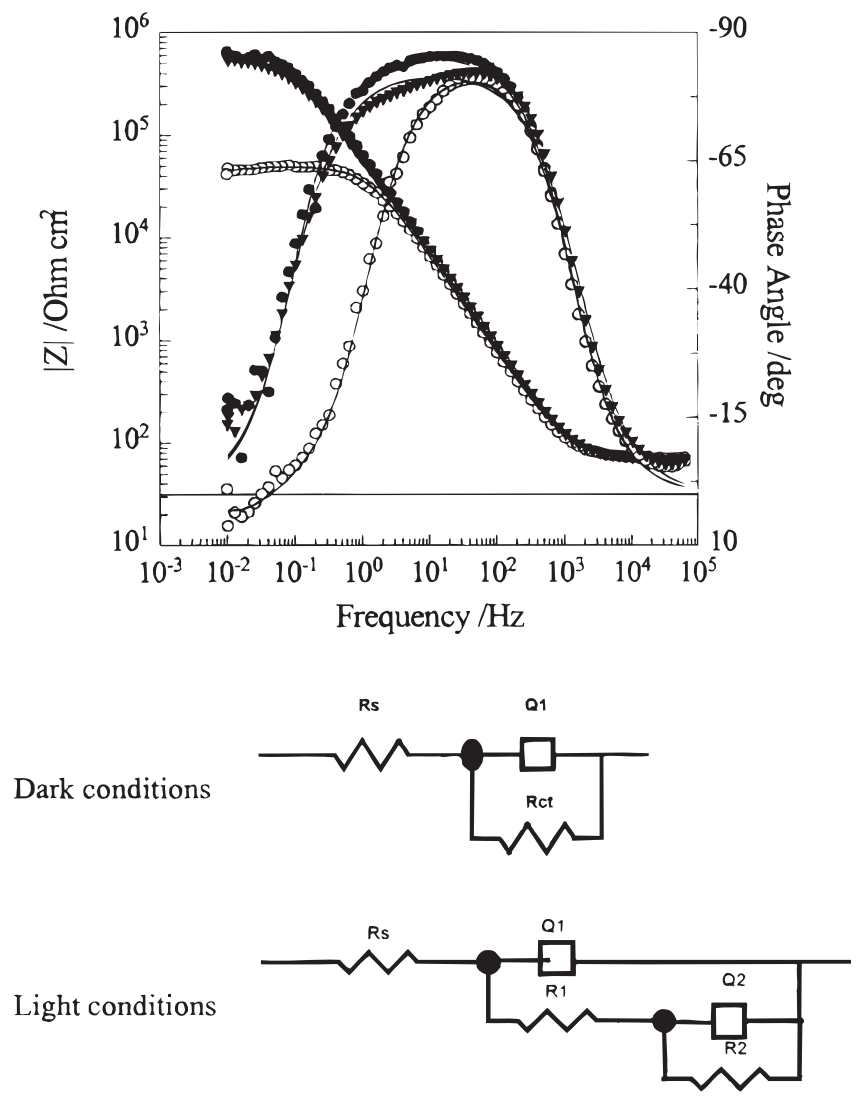

Figure 6. (a, top) Impedance data recorded under initial dark conditions ( $(\mathbf{O})$, followed by continuous illumination conditions $(\bigcirc)$, followed by dark conditions ( $\boldsymbol{\nabla}$ ) for Zn polarized at $800 \mathrm{mV}$ (SCE) in $\mathrm{pH}$ 9.2. (b) Equivalent circuits used in the fitting of impedance data.

surface was then illuminated and the impedance measured, and finally the light was removed and the impedance was remeasured under dark conditions. The equivalent circuits used in the fitting of these data are shown for the $\mathrm{pH} 13.0$ and 9.2 solutions in Fig. 5b and 6b, respectively. For the $\mathrm{pH} 13.0$ solution, a simple parallel RC circuit was used to fit the data recorded under illumination conditions, while the diffusion element, $Q_{2}$ was required for fitting of the data recorded under dark conditions. A simple RC circuit was used to fit the data recorded in the $\mathrm{pH} 9.2$ solution under dark conditions. But a more complex circuit, to account for the inductive behavior observed at low frequencies, was required to fit the data recorded under illumination conditions. Here a negative capacitor, $Q_{2}$, and a negative resistance element, $R_{2}$ were used. This circuit is based on a model used by Franceschetti and Macdonald, ${ }^{17}$ Armstrong, ${ }^{18}$ and Pettersson and Pound, ${ }^{19}$ where a negative resistance, $R_{2}$, and capacitance $C_{2}$ is used to model the inductive behavior as opposed to using very high inductances. This inductive behavior was observed only under illumination at high anodic potentials in this $\mathrm{pH} 9.2$ solution and always disappeared once the light stimulus was removed, Fig. 6a. The actual significance of this inductance is not clear, but it is consistent with the

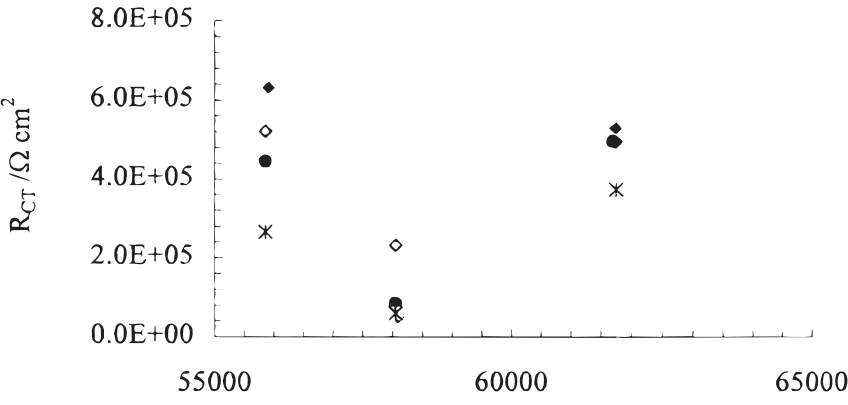

Polarisation Time /s

Figure 7. Charge-transfer resistance, $R_{\mathrm{CT}}$, plotted as a function of initial dark conditions, followed by illumination conditions, followed by final dark conditions for $\mathrm{Zn}$ polarized in $\mathrm{pH} 9.2$ solution at $800 \mathrm{mV}(\mathrm{SCE})(\diamond)$, $500 \mathrm{mV}(\mathrm{SCE})(\diamond)$, and $200 \mathrm{mV}(\mathrm{SCE})(\bullet)$, and in $\mathrm{pH} 10.3$ solution at $800 \mathrm{mV}(\mathrm{SCE})(\star)$.

oxidation current being impeded by an inductive current flowing in the opposite direction at the electrode/electrolyte interface. This may be associated with the formation and precipitation of a zinc-hydroxy layer, which can occur under these photoinduced dissolution conditions. However, as this inductive effect was seen only under this set of conditions it was explored no further.

The values for some of the common fitted parameters are shown in Table I. For the $\mathrm{pH} 9.2$ solution under illumination conditions, $R_{\mathrm{CT}}=R_{1}+R_{2}$, where $R_{2}$ is $-6.4 \times 10^{3} \Omega \mathrm{cm}^{2}$. It can be seen from this table that illumination decreases $R_{\mathrm{CT}}$ and leads to an increase in the capacitance, $Q_{1}$. These changes are very pronounced in the alkaline $\mathrm{pH} 13.0$ solution where $R_{\mathrm{CT}}$ under illumination conditions is only of the order of $1 \mathrm{k} \Omega \mathrm{cm}^{2}$. It is also clear from these data that polarization of zinc in the $\mathrm{pH} 9.2$ and 10.3 solutions leads to the formation of more stable passive films compared to that in the $\mathrm{pH} 13.0$ solution. This can be seen from both the charge-transfer resistance and double-layer capacitance values, Table I.

In Fig. 7 the charge-transfer resistance is plotted as a function of time for $\mathrm{Zn}$ polarized at potentials of 200, 500, and $800 \mathrm{mV}(\mathrm{SCE})$ in the $\mathrm{pH} 9.2$ solution and at $800 \mathrm{mV}(\mathrm{SCE})$ in the 10.3 solution. The points plotted at $56 \times 10^{3}$ s represent the data collected under dark conditions. The points plotted at $58 \times 10^{3} \mathrm{~s}$ represent the data collected under illumination conditions, while the data plotted at $62 \times$ $10^{3} \mathrm{~s}$ were obtained from the impedance data measured under dark conditions, but following the illumination period. These data illustrate, very clearly, that illumination results in a decrease in the chargetransfer resistance and that once the light source is removed that the charge-transfer resistance is restored to values comparable to those under the original dark conditions.

In order to gain information on the thickness of the passive layers formed in the presence and absence of illumination, capacitance measurements were performed on zinc polarized under dark and illumination conditions. These data were obtained by polarizing zinc at a scan rate of $5 \mathrm{mV} \mathrm{s}^{-1}$ from the open-circuit potential up to the potential where transpassive dissolution occurs. A $10 \mathrm{mV}$ perturbation signal at $1 \mathrm{kHz}$ was superimposed on this positive potential sweep and the imaginary impedance, from which the capacitance is calculated, measured as a function of the applied potential. Assuming

Table $\mathrm{I} . \boldsymbol{R}_{\mathrm{CT}}, Q_{1}$, and $n$ values calculated from impedance data for $\mathrm{Zn}$ polarized in $\mathrm{pH} 9.2,10.3$, and 13.0 solutions under conditions of illumination and nonillumination.

\begin{tabular}{|c|c|c|c|c|c|c|}
\hline \multirow[t]{2}{*}{ Parameter } & \multicolumn{2}{|c|}{ pH 9.2 solution } & \multicolumn{2}{|c|}{ pH 10.3 solution } & \multicolumn{2}{|c|}{ pH 13.0 solution } \\
\hline & Dark & Light & Dark & Light & Dark & Light \\
\hline$R_{\mathrm{CT}} / \Omega \mathrm{cm}^{2}$ & $6.31 \times 10^{5}$ & $4.30 \times 10^{4}$ & $2.65 \times 10^{5}$ & $5.94 \times 10^{4}$ & $1.20 \times 10^{4}$ & $1.30 \times 10^{3}$ \\
\hline$Q_{1} / \mu \mathrm{F} \mathrm{cm}^{-2}$ & 2.68 & 3.07 & 5.25 & 5.60 & 12.7 & 24.7 \\
\hline$n$ & 0.95 & 0.94 & 0.92 & 0.91 & 0.93 & 0.88 \\
\hline
\end{tabular}




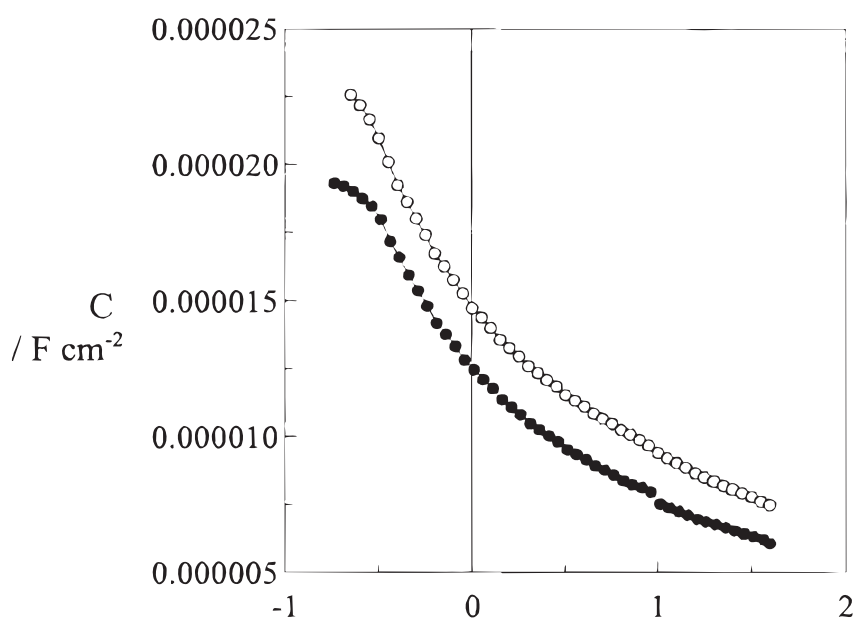

Potential / V(SCE)

Figure 8. Capacitance plotted as a function of potential for $\mathrm{Zn}$ polarized in $\mathrm{pH} 13.0$ solution under conditions of illumination $(\bigcirc)$ and nonillumination (๑).

that the passive layer behaves as a dielectric then the measured capacitance is proportional to the thickness of the passive layer, Eq. 1

$$
d=\frac{\epsilon \epsilon_{\mathrm{o}} A}{C}
$$

Here, $d$ is the thickness of the passive layer, $\epsilon_{0}$ is the permitivity of free space, and $\epsilon$ is the dielectric constant of the passive layer (8.5 for $\mathrm{ZnO}{ }^{20,21}$ ). In Fig. 8, the capacitance measured as a function of applied potential for zinc polarized in the $\mathrm{pH} 13.0$ solution in the dark and under illumination conditions is shown. It should be noted that these potentiodynamic conditions will not lead to the achievement of a steady state in the thickness of the passive films, however it is clear from these data that illumination of the surface results in a decrease in the thickness of the passive layers formed. Similar, data were obtained in the $\mathrm{pH} 9.2$ and 10.3 solutions.

Semiconducting properties. - In order to obtain information on the semiconducting properties of the films exposed to intermittent illumination, Mott-Schottky analyses were carried out for passive films formed in the $\mathrm{pH}$ 13.0, 10.3, and 9.2 solutions. In Fig. 9, representative Mott-Schottky plots recorded under dark conditions, for specimens previously exposed to continuous dark conditions and an intermittent illumination period are shown for zinc polarized in the $\mathrm{pH} 9.2$ solution. In these measurements, a passive film was first formed at $800 \mathrm{mV}(\mathrm{SCE})$ in the alkaline solution. In the case of the dark experiments, the passive film was formed under dark conditions for $17 \mathrm{~h}$ and then the Mott-Schottky data were recorded. In the case of the illumination experiments, the electrode was first passivated for $15.5 \mathrm{~h}$ under dark conditions, then illuminated for a 30 min period and finally polarized for a further $1 \mathrm{~h}$ period in the dark. In the measurements, the potential was swept from the formation potential [800 $\mathrm{mV}(\mathrm{SCE})]$ at a sweep rate of $0.01 \mathrm{~V} \mathrm{~s}^{-1}$ in the negative direction. Identical data were obtained on reversing the sweep back to the original formation volt-

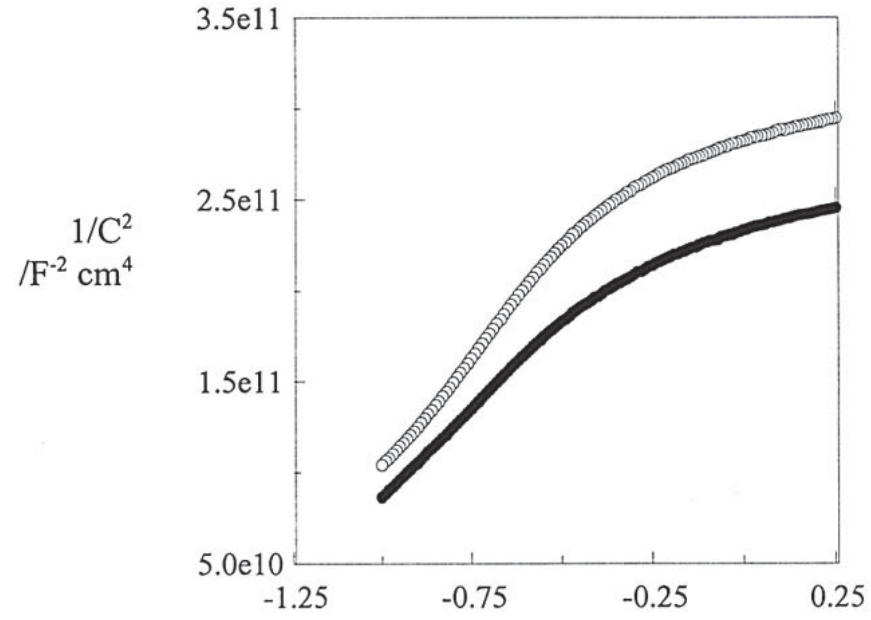

Potential /V(SCE)

Figure 9. Mott-Schottky plots for $\mathrm{Zn}$ polarized in $\mathrm{pH} 9.2$ solution following prior illumination $(\bigcirc)$ and dark conditions $(\mathbf{O})$.

age and with different scan rates. This shows that the film thickness and the donor densities remain constant throughout the experiment. The total capacitance, $C$, which consists of contributions from the space-charge capacitance and the Helmholtz capacitance, was calculated from the impedance data. In this case, the space-charge capacitance was calculated by correcting this measured capacitance data for Helmholtz contributions using a conservative value of $20 \mu \mathrm{F} \mathrm{cm}^{-2}$ for the Helmholtz capacitance. The potential dependence of $1 / C_{\mathrm{sc}}^{2}$ follows the Mott-Schottky equation 22,23

$$
\frac{1}{C_{\mathrm{SC}}^{2}}=\frac{2}{q \epsilon \epsilon_{\mathrm{o}} N_{\mathrm{D}}}\left(E-E_{\mathrm{FB}}-\frac{k T}{q}\right)
$$

where $N_{\mathrm{D}}$ is the donor density, $E_{\mathrm{FB}}$ is the flatband potential, $\epsilon$ is the dielectric constant of the semiconductor film (i.e. 8.5 for $\mathrm{ZnO}^{20,21}$ ), $\epsilon_{0}$ is the permittivity of free space and q is the electronic charge. The Mott-Shottky plots shown in Fig. 9, exhibit well-defined linear portions over a relatively wide potential region, and are characteristic of those for an n-type semiconductor, in agreement with other publications. ${ }^{2,6}$ The donor densities, calculated from the slopes of the linear regions of the plot were found to be about $1.0 \pm 0.2 \times 10^{20} \mathrm{~cm}^{-3}$ for the electrodes under continuous dark conditions and 7.5 $\pm 2.0 \times$ $10^{19} \mathrm{~cm}^{-3}$ for the electrodes exposed to prior illumination. The flatband potentials were calculated as $-1.42 \pm 0.30 \mathrm{~V}(\mathrm{SCE})$ for the illuminated electrode and $-1.38 \pm 0.30 \mathrm{~V}$ (SCE) for the dark electrode. The donor densities calculated here are in good agreement with those calculated previously ${ }^{2,6}$ which range between $1 \times 10^{20}$ and $3 \times 10^{20} \mathrm{~cm}^{-3}$. Similar photoinduced modifications in the donor densities were found for the other electrolytes. These results are summarized in Table II. Here it can be seen that although the decrease in the donor densities is not far removed from the experimental error, $\pm 0.2 \times 10^{20} \mathrm{~cm}^{-3}$, that consistently lower donor densities are found for the electrodes, regardless of the $\mathrm{pH}$ of the solution, subjected to illumination conditions. The relatively high donor

Table II. $N_{\mathrm{D}}$ and $E_{\mathrm{FB}}$ calculated from Mott-Schottky analyses for $\mathrm{Zn}$ polarized in pH 9.2, 10.3, and 13.0 solutions under conditions of prior

\begin{tabular}{|c|c|c|c|c|c|c|}
\hline \multirow[t]{2}{*}{ Parameter } & \multicolumn{2}{|c|}{ pH 9.2 solution } & \multicolumn{2}{|c|}{ pH 10.3 solution } & \multicolumn{2}{|c|}{$\mathrm{pH} 13.0$ solution } \\
\hline & Dark & Light & Dark & Light & Dark & Light \\
\hline $\begin{array}{l}\mathrm{N}_{\mathrm{D}} / 10^{20} \mathrm{~cm}^{-3} \\
E_{\mathrm{FB}} / \mathrm{mV} \text { (SCE) }\end{array}$ & $\begin{array}{c}1.0 \\
-1380\end{array}$ & $\begin{aligned} & 0.75 \\
- & 1420\end{aligned}$ & $\begin{array}{r}4.0 \\
-1100\end{array}$ & $\begin{array}{r}1.6 \\
-1160\end{array}$ & $\begin{array}{r}7.3 \\
-760\end{array}$ & $\begin{array}{r}5.2 \\
-800\end{array}$ \\
\hline
\end{tabular}
illumination and nonillumination. 
densities obtained for the alkaline $\mathrm{pH} 13.0$ solution reflect the greater defect structure of the films formed in this alkaline solution. The flatband potentials tend to become more negative following illumination. This is probably due to the photoinduced corrosion of the surface and is consistent with the reports of Dewald ${ }^{24}$ who showed that the flatband potentials varied with time, becoming more negative, after etching of $\mathrm{ZnO}$ in $\mathrm{H}_{3} \mathrm{PO}_{4}$. This gradual change was attributed to slow dissolution or corrosion of the surface leading to a new surface condition. The values of the flatband potentials in the $\mathrm{pH}$ 13.0 solutions agree well with those of Bohe et al. ${ }^{6}$ However, the values calculated for the other solutions in which the electrodes were prepassivated for $17 \mathrm{~h}$ are considerably more negative than those reported by Bohe et al. ${ }^{6}$ This seems to be associated with the longer passivation periods used in this study.

A plot of the donor densities as a function of the formation potential for zinc polarized under dark conditions and subjected to the previously described intermittent illumination conditions in the $\mathrm{pH} 9.2$ solution is shown in Fig. 10. It can be seen from this figure that the donor densities decrease with an increase in the formation potential, in agreement with other reports, ${ }^{2}$ but, again it is seen, that at each formation potential, prior illumination leads to a decrease in the donor densities.

\section{Discussion}

The experimental results presented here show that dissolution of the passive film on zinc occurs under illumination conditions. This can be seen clearly from the anodic polarization data presented in Fig. 1, the current-time transient data presented in Fig. 2 and the complex impedance data presented in Fig. 4, 5, and 6. These photoinduced dissolution effects can be explained in terms of the photoinduced dissolution of $\mathrm{ZnO}$ which is well known to be present in the passive films grown on polycrystalline $\mathrm{Zn} .{ }^{25,26}$ It is well known that $\mathrm{ZnO}$ is a highly-doped n-type defect semiconductor with the principal defect being the zinc interstitial. ${ }^{2,6}$ On illumination of $\mathrm{ZnO}$ with sufficiently energetic photons, electrons are promoted from the valence to the conduction band, generating electron-hole pairs. These electron-hole pairs separate due to band bending. The holes are transported to the film-solution interface where they undergo an electrochemical reaction with some suitable redox species. In the absence of any suitable redox species in solution, as in this study, the thermodynamically favored reaction of the generated holes is the reaction with $\mathrm{ZnO}$ leading to the photodecomposition of $\mathrm{ZnO}^{9}$

$$
\mathrm{ZnO}+2 p^{+}+n \mathrm{OH}^{-} \rightarrow \mathrm{Zn}(\mathrm{OH})_{n}^{(2-n)}+\frac{1}{2} \mathrm{O}_{2}
$$

The nature of these soluble corrosion species $\mathrm{Zn}(\mathrm{OH})_{n}^{(2-n)}$ will depend on the $\mathrm{pH}$ of the solution. In the case of these alkaline solutions the likely corrosion products ${ }^{27}$ are $\mathrm{Zn}(\mathrm{OH})_{4}^{2-}$ or $\mathrm{Zn}(\mathrm{OH})_{3}^{-}$. But, in any case, this photoinduced dissolution leads to the destruction of the anodically formed passive film.

It can be seen from the capacitance-voltage data presented in Fig. 8 that this photoinduced dissolution is accompanied by a decrease in the oxide film thickness, as expected. Indeed, this can be seen further from the capacitance data presented in Table I. These capacitance data, in the case of the $\mathrm{pH} 9.2$ and 10.3 solutions, were measured after a $15 \mathrm{~h}$ period and thus reflect steady-state conditions. Using the well-known parallel plate expression for the capacitance, $C$, to estimate the thickness of the passive layer, Eq. 1, it can be seen that in all cases lower values of film thickness are estimated for the illuminated specimens. This is consistent with the photodecomposition of the passive layer and a resulting decrease in the thickness of the passive layer.

The enhanced dissolution in the $\mathrm{pH} 9.2$ and 10.3 solutions can be explained in terms of this photoinduced decomposition of the passive layer. The increase in the photocurrents with higher anodic bias, Fig. 3a, is consistent with the rise in the concentration of surface holes, which increases with increasing surface potential barrier. However, in the case of the $\mathrm{pH} 13.0$ solution, the photocurrents in the region of $20 \mu \mathrm{A} \mathrm{cm}{ }^{-2}$ and increasing to in excess of $30 \mu \mathrm{A}$ $\mathrm{cm}^{-2}$ over a 30 min illumination period, Fig. 2b, are not consistent with simply a photoinduced dissolution reaction. It can be seen from Fig. $3 b$ that the photocurrents increase with increasing film formation period, as expected, due to the growth of the oxide and increase in the width of the space-charge layer. However, in the case of the $\mathrm{pH} 13.0$ solution, the increasing anodic photocurrent combined with the relatively low thickness of the passive film in this solution (the capacitance being of the order of $25 \mu \mathrm{F} \mathrm{cm}{ }^{-2}$ ), strongly point to a photoinduced dissolution reaction accompanied by an electrochemical dark anodic reaction. The very thin oxide, which exists under these illumination conditions, is not sufficiently protective to enable passivation of the electrode and an accompanying dark current flows. In addition, the oxide formed, through a dissolution-repassivation process, in this $\mathrm{pH} 13.0$ solution is more porous in nature than those formed in the less alkaline solutions. This may also contribute to the enhanced dissolution of the passive layers formed in this solution on illumination. Indeed, when the light is switched off it takes some time for the electrode to repassivate in the $\mathrm{pH} 13.0$ solution and to a lesser extent in the $\mathrm{pH} 10.3$ solution. This $\mathrm{pH}$ dependence on the dissolution rate is in agreement with the data of Futsuhara et al. ${ }^{13}$ These authors found, by monitoring the dissolution rate of $\mathrm{ZnO}$ thin films using the zinc content in the film before and after the photodissolution reaction, that the optimum $\mathrm{pH}$, in the interval 7.0 to 12.0, for the photodissolution of $\mathrm{ZnO}$ was 12.0.

Although this photoinduced dissolution reaction only occurs while the specimens are illuminated, there is some evidence to suggest that the prior illumination procedure leads to some modifications of the passive layers. This is evident from the Mott-Schotty analyses, Fig. 9 and 10, where prior illumination of the specimens is seen to give rise to a decrease in the donor densities. As these tests were recorded following a further hour after the photoinduced dissolution process, to ensure the complete repassivation of the electrode and to ensure similar oxide film thickness, it seems that the photoinduced excitation of the $\mathrm{ZnO}$ semiconductor and/or the dissolution/repassivation events induced by the illumination procedure leads to a true decrease in the defect structure. The only other parameter that may affect the validity of these results are slight changes in the actual surface area following the illumination procedure. However if such a change in the surface area did occur then the true donor densities observed under illumination would in fact be even smaller that the values quoted here.

\section{Conclusions}

Significant dissolution of anodically formed passive layers on zinc was observed in alkaline $\mathrm{pH}$ 9.2, 10.3, and 13.0 solutions on illumination with polychromatic light. This was attributed to the photodecomposition of $\mathrm{ZnO}$, which occurs through reaction with surface holes generated during the illumination period. This photoinduced dissolution effect only persisted while the electrodes were

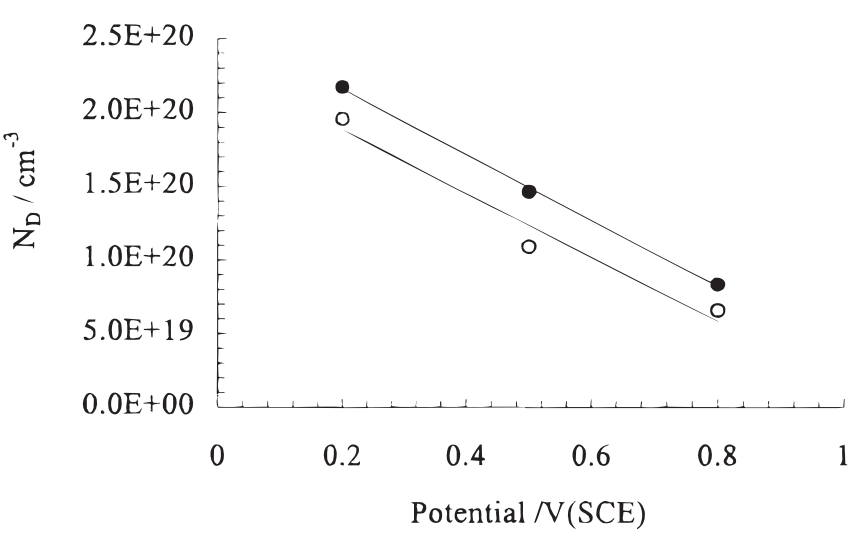

Figure 10. Donor densities plotted as a function of the formation potential for $\mathrm{Zn}$ polarized in $\mathrm{pH} 9.2$ solution under conditions of prior illumination (○) and dark conditions (O). 
illuminated. However, prior illumination of the anodic passive films gave rise to a decrease in the donor densities.

\section{Acknowledgments}

The authors gratefully acknowledge the support of this work by Enterprise Ireland, under the Basic Science Research Grants Award, Project Code SC/96/456.

The National University of Ireland, Maynooth assisted in meeting the publication costs of this article.

\section{References}

1. T. E. Graedel, J. Electrochem. Soc., 136, 193 (1989).

2. D. D. Macdonald, K. M. Ismail, and E. Sikora, J. Electrochem. Soc., 145, 3141 (1998).

3. J. Buchholz, Surf. Sci., 101, 146 (1980).

4. T. Burleigh, Corrosion, 45, 464 (1989).

5. P. Scholl, X. Shan, D. Bonham, and G. A. Prentice, J. Electrochem. Soc., 138, 895 (1991).

6. A. E. Bohe, J. R. Vilche, K. Juttner, W. J. Lorenz, W. Kautek, and W. Paatsch, Corros. Sci., 32, 621 (1991)

7. G. Heiland, E. Mollwo, and F. Stockmann, Solid State Phys., 8, 191 (1959).

8. A. K. Vigh, in Electrochemistry of Metals and Semiconductors, Marcel Dekker, New York (1973)
9. H. Gerischer, J. Electrochem. Soc., 113, 1174 (1966).

10. H. Gerischer, J. Electroanal. Chem., 82, 133 (1977).

11. J. Domenech and A. Prierto, J. Phys Chem., 90, 1123 (1986).

12. M. Okano, K. Itoh, A. Fujishima, and K. Honda, J. Electrochem. Soc., 134, 837 (1987).

13. M. Futsuhara, K. Yoshioka, Y. Ishida, O. Takai, K. Hashimoto, and A. Fujishima, J. Electrochem. Soc., 143, 3743 (1996).

14. E. Juzeliunas, P. Kalinauskas, A. Stankeviciute, A. Sudavicius, and A. Survila, Corrosion, 51, 673 (1995).

15. P. Spathis and I. Poulios, Corros. Sci., 37, 673 (1995)

16. J. R. Macdonald, Impedance Spectroscopy, Wiley, New York (1987).

17. D. R. Franceschetti and J. R. Macdonald, J. Electroanal. Chem., 82, 271 (1977).

18. D. Armstrong, J. Electroanal. Chem., 34, 387 (1972).

19. R. F. A. Jargelius-Pettersson and B. G. Pound, J. Electrochem. Soc., 145, 1462 (1998).

20. N. Sato and K. Kudo, Electrochim. Acta, 16, 447 (1971)

21. S. R. Morrison, Electrochemisty of Semiconductos and Oxidized Metal Electrodes, Plenum Press, New York (1980).

22. N. F. Mott, Proc. R. Soc. London Ser. A, 171, 27 (1939).

23. W. Schottky, Z. Phys., 113, 367 (1939).

24. J. F. Dewalt, Bell Syst. Tech. J., 39, 615 (1960).

25. S. T. Mayer and R. H. Muller, Abstract 511, p. 769, The Electrochemical Society Extended Abstracts, Vol. 90-1, Montreal, Quebec, Canada, May 6-11, 1990.

26. R. D. Armstrong and M. F. Bell, in Special Periodical Reports on Electrochemistry, Vol. 4, H. R. Thirsk, Editor, p. 1, The Chemical Society, London (1974).

27. R. A. Reichle, K. G. Mc Curdy, and L. G. Hepler, Can. J. Chem., 53, 3841 (1975). 